\title{
Decomposition of xylene in strong ionization non-thermal plasma at atmospheric pressure
}

\author{
Yuan $\mathrm{Jia}^{1}$, Chengwu Yi ${ }^{1, *}$, Rongjie Yi ${ }^{1, *}$, Miao Jiang ${ }^{1}$, Baolin Zhang ${ }^{2}$,Qi Zhang ${ }^{1}$ \\ ${ }^{1}$ School of the Environment and Safety Engineering, Jiangsu University, 212013, Zhenjiang, China \\ ${ }^{2}$ Zhenjiang ecological environment science and technology consulting center, 212001, zhenjiang, China
}

\begin{abstract}
A large amount of volatile organic compounds (VOCs) produced by industry have caused serious environmental pollution. In this paper, the removal effect of simulated xylene by strong ionization dielectric barrier discharge (DBD) plasma at atmospheric pressure and its degradation mechanism and pathway were studied. The effect of gas residence time, and initial xylene concentration was studied. The results showed that higher voltage caused an increase in discharge power, and with the increase of voltage, the concentration of ozone and nitrogen oxide in the reactor increased. The degradation efficiency decreased from $98.1 \%$ to $80.2 \%$ when xylene concentration increased from $50 \mathrm{ppm}$ to $550 \mathrm{ppm}$ at $4 \mathrm{kV}$. And with the increase of residence time from $0.301 \mathrm{~s}$ to $1 \mathrm{~s}$, the degradation efficiency increased from $78.5 \%$ to $98.6 \%$. According to GC-MS analysis, the degradation products were ethyl acetate and n-hexylmethylamine at $4 \mathrm{kv}$. And the main intermediates are 2,4-2-tert-butylphenol, 2-aminopentane, 2-methyl-5 - (2-aminopropyl) - phenol and propionamide at 1.5kV.
\end{abstract}

\section{Introduction}

Due to the increase of factory emissions and the decrease of green vegetation, there are more and more VOCs in the air. VOCs are very harmful to human health and environment. VOCs can also stimulate the respiratory tract, kidney, lung, liver, digestive system, nervous system and hematopoietic system, and cause pathological changes. and Some VOCs are toxic, irritant, teratogenic and carcinogenic.

Most VOCs have photochemical reactivity. Under the sunlight, VOCs will react with NOx in the atmosphere to produce ozone, free radicals and other strong oxidizing secondary pollutants, thus producing photochemical $\operatorname{smog}^{[1]}$.

The reaction results in the formation of tropospheric ozone, depletion of stratospheric ozone, secondary organic aerosols, and the absorption of thermal infrared, thus aggravating global warming.

Common VOC removal methods include adsorption, absorption, membrane separation, condensation, biodegradation, thermal and catalytic combustion, and photocatalysis. However, these methods are not good for the treatment of VOCs with low concentration, and it is easy to produce secondary pollution.

Non-thermal plasma (NTP) technology has become popular as a promising technology adaptable to room temperature and atmospheric pressure. Compared with other VOCs treatment technologies, NTP technology has the following advantages: it has low environmental requirements; the operation cost is low; it is widely used and has good treatment effect on almost all VOCs.

To achieve generate NTP at atmospheric pressure or above atmospheric pressure, the strong ionization discharge is a good choice. The electric field strength is greater than $400 \mathrm{Td}$, the average energy of electrons is greater than $10 \mathrm{eV}$, and electron density is higher than $10^{15}$ $\mathrm{cm}^{-3}[2,3]$.

The purpose of this study was to investigate the operation of a strong ionization DBD to remove xylene from a synthetic polluted air stream. Experimental evaluations included the effect of, initial concentration, gas residence time, and other relevant parameters. Production of ozone and $\mathrm{NO}_{\mathrm{x}}$ by-products were also investigated. In addition, the oxidation by-products were analysed by GCMS, and a suggested xylene decomposition pathway was discussed.

\section{Materials and methods}

\subsection{Experimental set-up}

A schematic representation of the whole experimental set up to study the decomposition of xylene is shown in Figure 1. The set-up has three main components: (1) the gas flow component comprising of the air pump and nitrogen and oxygen gas supply tanks under flow control, (2) the reactor component comprising of the strong ionization DBD reactor, high-voltage AC supply, and (3) the analysis component comprising of the IQ1000 VOCs concentration detector, CENTER314 temperature and humidity meter, WaveJet 312A oscilloscope, Agilent6890-59758 GCMS, NOVA5003-S flue gas analyzer, and a computer. 


\subsection{The reactor system}

frequency power supply. The reactor is a rectangular box, $227 \mathrm{~mm} \times 145 \mathrm{~mm} \times 22 \mathrm{~mm}$ in dimension. The shell of the reactor is made of cast iron, and the shell is a grounding electrode. A layer of dense $\alpha-\mathrm{Al} 2 \mathrm{O} 3$ is used as dielectric in the upper and lower shells. A piece of sintered metal silver is used as the discharge electrode in the middle of the reactor. The outside of the reactor is equipped with black heat sink. The cooling fan is installed near the reactor to prevent the reactor from overheating. The discharge electrode is made of sintered metal silver. The actual figure is shown in Figure 1.

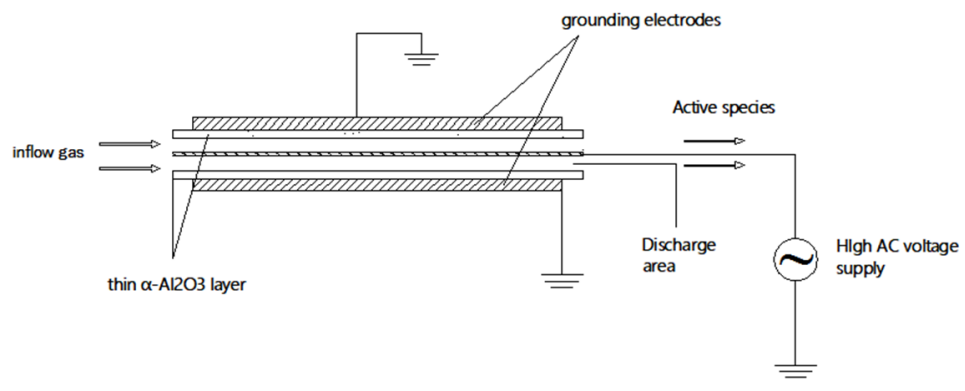

Figure 1. Schematic representation of the strong ionization DBD reactor

\subsection{Experimental process}

The experimental process is shown in the figure 2 .

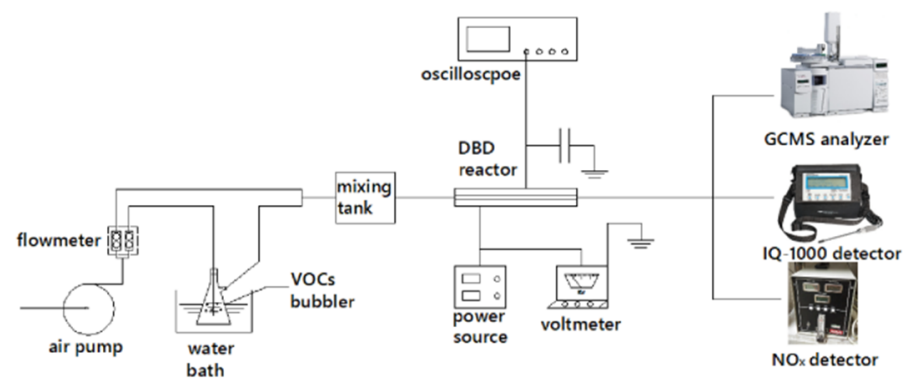

Figure 2.Experimental flow chart.

\section{Results and discussion}

According to $\mathrm{Kim}^{[4]}$, there are two main reaction mechanism schemes leading to the decomposition process of xylene in the strong ionization discharge chamber. Firstly, high-energy electrons generated within the discharge chamber may attack xylene directly, leading to the removal of xylene from the polluted gas stream (direct electron attack). Secondly, high chemically reactive gas-phase radicals such as $\bullet \mathrm{OH}$ (hydroxyl), $\mathrm{HO}_{2}$ (hydroperoxyl), and $\mathrm{O}$ (oxygen atom) can also react with xylene to form new environmentally less toxic by-products (indirect gas-phase radical reaction).

\subsection{Effect of initial xylene concentration}

Figure 3 shows the impact of initial xylene concentration on removal efficiency for different voltage $(1.5 \mathrm{kV}, 2.0 \mathrm{kV}$, $3.0 \mathrm{kV}, 4 \mathrm{kV})$. The initial concentration is varied between 200 and $600 \mathrm{ppm}$ with a total gas flow rate of $5 \mathrm{~L} / \mathrm{min}$. The removal efficiency of xylene pollutant is significantly dependent on the initial concentration of formaldehyde as well as the supply voltage.

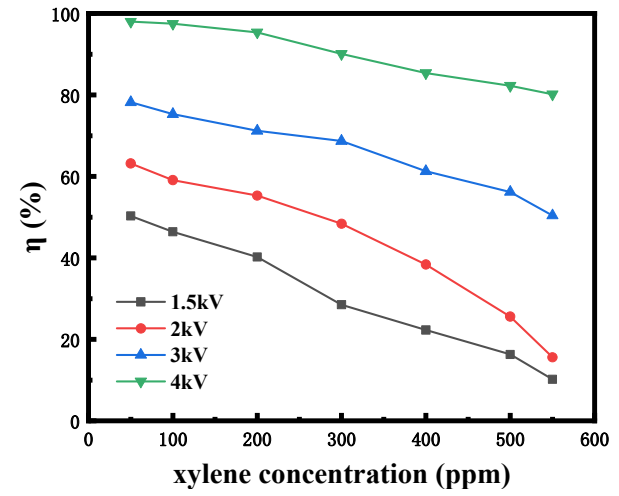

Figure 3. effect of initial concentration on xylene degradation efficiency

At constant supply voltage, removal efficiency decreases for increasing initial xylene concentration. For example, at $4 \mathrm{kV}$, the removal efficiency reaches $98.1 \%$ when the initial concentration is $50 \mathrm{ppm}$, but decreases to $80.2 \%$ when initial concentration is $550 \mathrm{ppm}$. When the gas flow is constant, the amount of reactive species, high-energy electrons, and active species generated by plasma could be same at a certain operating condition. The number of residual xylene molecules increase with increasing the concentration of xylene. Consequently, the 
removal efficiency of xylene decreases for increasing initial xylene concentration.

\subsection{Effect of gas residence time}

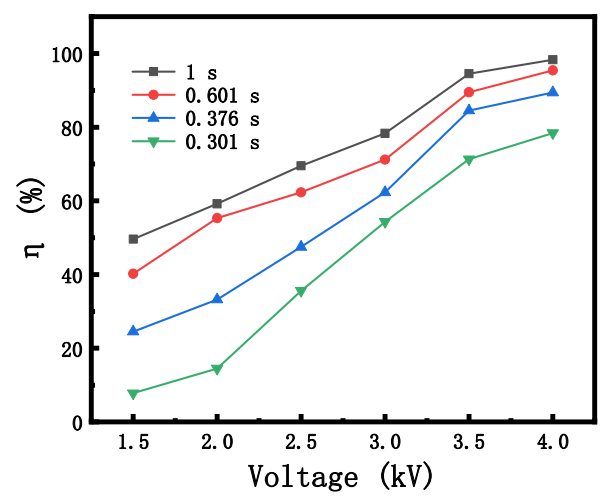

Figure 4 effect of spatial velocity on xylene degradation. Efficiency.

The gas residence time is the ratio of the discharge reactor volume to the gas mass flow rate ${ }^{[5]}$. Figure 4 shows that at any supply voltage xylene removal efficiency increased with increasing gas residence time from 0.301 to $1 \mathrm{~s}$. Apparently, xylene removal is significantly dependent on the gas residence time. The xylene removal efficiency was 98.6, 95.4, 89.4 and $78.5 \%$ when the gas residence time was $1,0.601,0.376$ and $0.301 \mathrm{~s}$ at $4.0 \mathrm{kV}$, respectively. This agrees with the observation of Hongxiang et $\mathrm{al}^{[6]}$.

The possible reason for this phenomenon is that, with the decrease of gas residence time, the reaction gas decrease and the reaction time shorten. Although the chances of inelastic collision of gas molecules with free radicals and energetic electrons can increase, more gas molecules pass directly through the reactor without any interaction with free radicals and electrons because of the shortened residence time.

\subsection{Production of ozone (O3) and NOx}

Ozone is an important inorganic by-product in plasma induced degradation of organic pollutants. Figure 5 shows the ozone produced by the strong ionization DBD under different voltage. As shown in the figure, $\mathrm{O}_{3}$ production increases with the increase of applied voltage. When the voltage is less than $3 \mathrm{kV}$, the growth rate of $\mathrm{O}_{3}$ production gradually becomes faster, while when the voltage is greater than $3 \mathrm{kV}$, the growth rate of $\mathrm{O}_{3}$ production becomes slow.. This is consistent with the observation of
Jiang et $\mathrm{al}^{[7]}$.

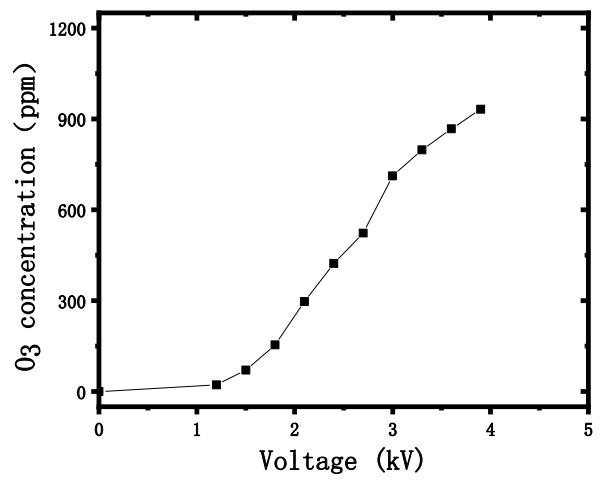

Figure 5 relationship between $\mathrm{O}_{3}$ and applied voltage.

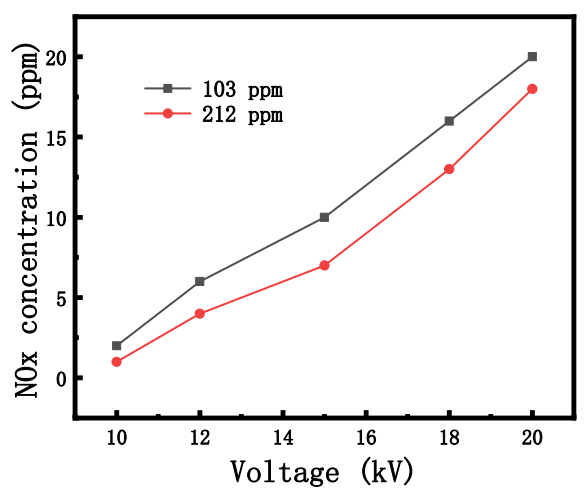

Figure $6 \mathrm{NO}_{\mathrm{x}}$ production in reaction products under different applied voltage.

NOx is another important inorganic by-product of DBD reactor, predominantly resulting from nitrogen and oxygen in the air used as carrier gas. Under the experimental conditions: the initial concentration of xylene is 103 ppm and $212 \mathrm{ppm}$, and the gas flow rate is $10 \mathrm{~L} / \mathrm{min}$, the change trend of NOx production with voltage is investigated. The results are shown in Figure 6. With the increase of voltage, NOx production in tail gas also increases. And it can be seen that the concentration of NOx decreases with the increase of xylene concentration. Guo et al[8] have shown a series of possible reactions leading to the formation and oxidation of NOx species during a DBD-catalyst process.

\subsection{Analysis of organic by-products}

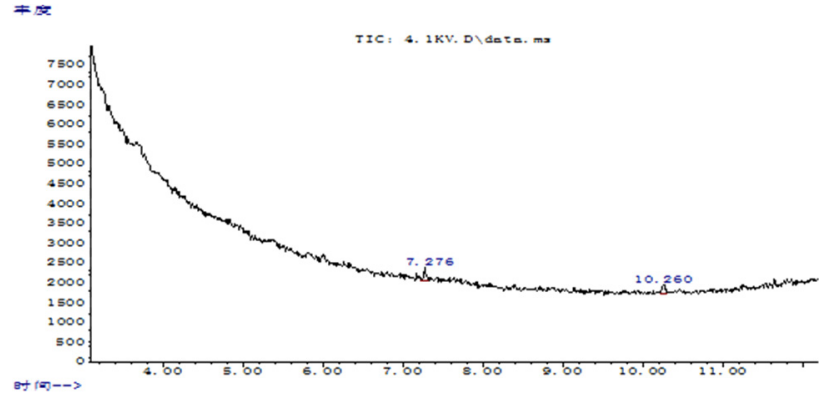

Figure 7 GC-MS analysis of xylene degradation products at $4.1 \mathrm{kV}$. 
The experimental conditions were as follows: voltage 4.1 $\mathrm{kV}$, discharge frequency $5.25 \mathrm{kHz}$, initial concentration $550 \mathrm{ppm}$, gas flow rate $10 \mathrm{~L} / \mathrm{min}$, temperature $30{ }^{\circ} \mathrm{C}$, relative humidity $81.2 \%$. The obtained GC-MS analysis chart is shown in Figure 7. It can be seen from the graph that there are only two very small peaks except dichloromethane, which is used as solvent. Through the library search, these two peaks represent ethyl acetate and n-hexylmethylamine respectively. The appearance of these two substances indicates that the benzene ring of xylene has been released after being treated by strong ionization discharge device and decomposed into small molecular organic matter which is easier to be degraded. The source of amino group in n-hexylmethylamine in the detected product may be NOx produced after nitrogen in air is ionized.

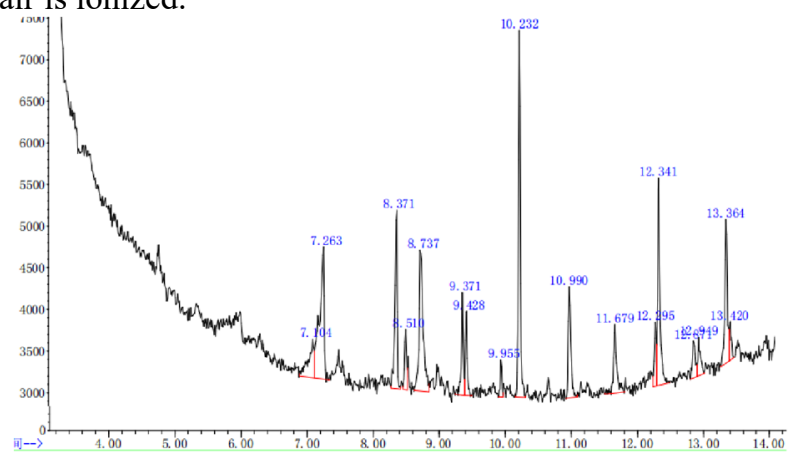

Figure 8 GC-MS analysis of xylene degradation products at $1.5 \mathrm{kV}$

It is difficult to determine the reaction path of degradation of xylene because of the low concentration and few kinds of products at high voltage. Therefore, changed voltage to $1.5 \mathrm{kV}$. The gas chromatogram of xylene degradation products is shown in Figure 8. The obtained GC-MS spectra are shown in Figure 8. By searching the spectrum library, it was determined that the main intermediate product was 2,4-2-tert-butylphenol, 2-aminopentane, Propionamide and 2-methyl-5-(2-aminopropyl)-phenol.

\subsection{Decomposition mechanism of xylene}

The dissociation energies of each chemical bond in xylene are $\mathrm{C}=\mathrm{C}$ is $5.5 \mathrm{eV}, \mathrm{C}-\mathrm{H}$ on methyl is $3.89 \mathrm{eV}, \mathrm{C}-\mathrm{H}$ on benzene ring is $4.93 \mathrm{eV}, \mathrm{C}-\mathrm{C}$ is $3.6 \mathrm{eV}$. Eq $1 \sim 4$ are the possible reaction equations in the reaction process.

$$
\begin{aligned}
& \mathrm{C}_{6} \mathrm{H}_{6} \mathrm{CH}_{3} \mathrm{CH}_{3}+e \rightarrow \mathrm{C}_{6} \mathrm{H}_{6} \mathrm{CH}_{3} \bullet+\mathrm{CH}_{3} \bullet \\
& \mathrm{C}_{6} \mathrm{H}_{6} \mathrm{CH}_{3} \mathrm{CH}_{3}+2 e \rightarrow \mathrm{C}_{6} \mathrm{H}_{6} \bullet+2 \mathrm{CH}_{3} \bullet \\
& \mathrm{C}_{6} \mathrm{H}_{6} \bullet+e \rightarrow \bullet \mathrm{C}_{6} \mathrm{H}_{6} \bullet \\
& \bullet \mathrm{C}_{6} \mathrm{H}_{6} \bullet+e \rightarrow \bullet \mathrm{C}_{4} \mathrm{H}_{4} \bullet+\bullet \mathrm{C}_{2} \mathrm{H}_{2} \bullet
\end{aligned}
$$

According to the 2-methyl-5-(2-aminopropyl)-phenol and 2,4-2-tert-butylphenol produced in the product, it can be seen that the methyl molecule on the xylene molecule is first separated. However, due to the lower high-energy electron concentration, a part of the $\cdot \mathrm{C} 6 \mathrm{H} 4 \cdot$ can not be further decomposed, and the original position of the two methyl groups will be replaced by other groups, which can prove the correctness of the above inference. However, hydroxyl groups are added to the benzene ring of the two products, and the activity of phenols is higher than that of benzene and xylene, and the ring opening reaction is more likely to occur.

Through the above analysis, the possible reaction pathway of xylene degradation in strong ionization discharge reactor is obtained, as shown in Figure 8.

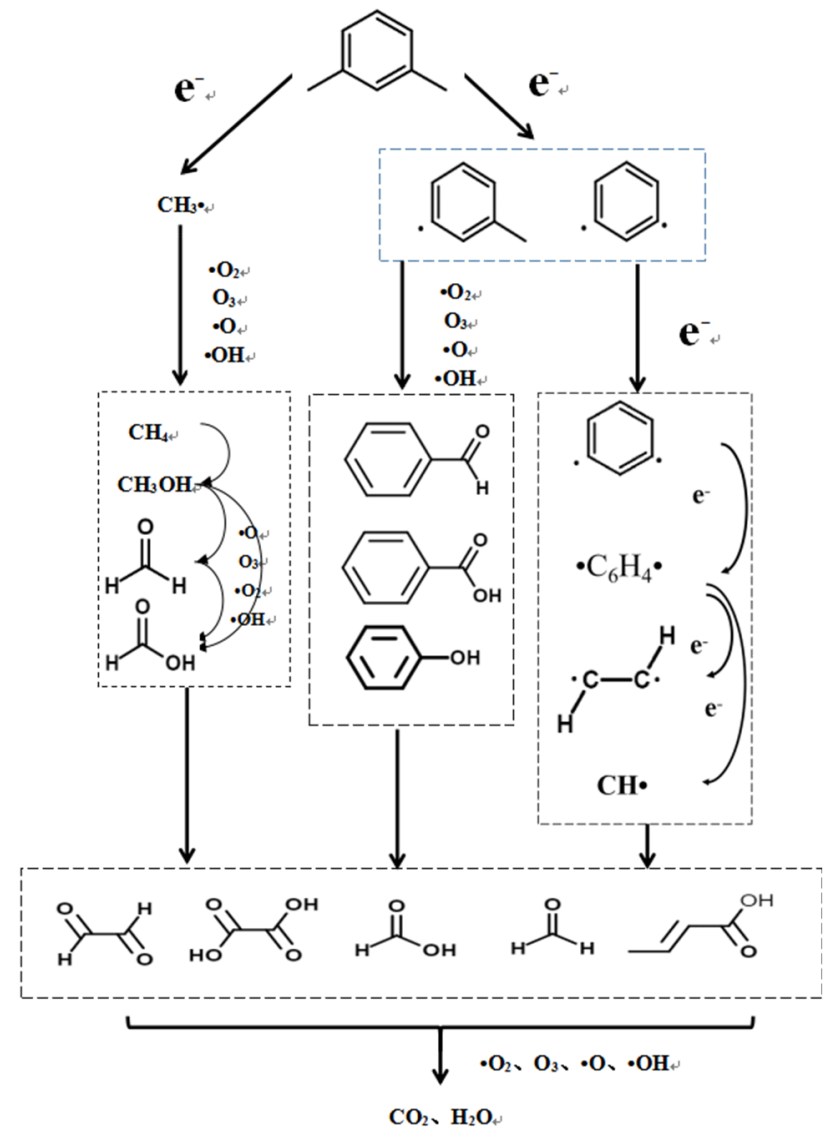

Figure 9 possible degradation pathways of p-xylene

\section{Conclusion}

In this study, a laboratory-scale strong ionization DBD reactor was constructed to generate high concentration $\cdot \mathrm{O}$, $\cdot \mathrm{OH}, \mathrm{O}_{3}$ and other active particles that were used to efficiently remove xylene from a synthetic polluted air stream at atmospheric pressure. In the plasma system, high-energy electrons and the active particles generated attack the organic pollutant to form $\mathrm{CO}_{2}$ and $\mathrm{H}_{2} \mathrm{O}$. Evaluations included, initial concentration, and space velocity (residence time). The experimental results revealed that $\mathrm{NO}_{\mathrm{x}}$ concentration increased with voltage. The degradation efficiency decreased from $98.1 \%$ to $80.2 \%$ when xylene concentration increased from $50 \mathrm{ppm}$ to $550 \mathrm{ppm}$ at $4 \mathrm{kV}$. In addition, it was realized that reducing residence time to $0.301 \mathrm{~s}$ although increased the number of inelastic collisions, leading to lower removal efficiency. By-product analysis was used to propose degradation pathway of xylene. The study provided a theoretical and experimental basis for the degradation of the organic pollutant from air stream by strong ionization discharge, with impressive removal efficiency. The experiment is relevant for air pollution prevention and 
control.

\section{Acknowledgements}

This work is supported by the Science and Technology Support Project Plan and Social Development of Zhenjiang city, China (Grant No. SH2012013).

\section{References}

1. Khan F I. Ghoshal A K. Removal of Volatile Organic Compounds from polluted air[J]. Journal of Loss Prevention in the Process Industries, 13(6): 527-545. (2000).

2. Zhang H. Fengsen Z. Xiaodong L. Changming D Dynamic behavior of a rotating gliding arc plasma in nitrogen: effects of gas flow rate and operating current. Plasma Sci Technology 19(4): 045401. (2017)

3. Manley T. The electric characteristics of the ozonator discharge. Trans Electrochem Soc 84(1): 83-96. (1943)

4. Kim H H. Nonthermal Plasma Processing for AirPollution Control: A Historical Review, Current Issues, and Future Prospects[J]. Plasma Processes \& Polymers. 1(2): 91-110. (2004).

5. Zhang H. Li K. Sun T. Jia J. Yang X. Shen Y. Wang J. Lou Z. The removal of styrene using a dielectric barrier discharge (DBD) reactor and the analysis of the by-products and intermediates. Res Chem Intermed 39(3): 1021-1035. (2013)

6. Hongxiang O. Chengwu Y. Wenming Q. et al. Study on formaldehyde degradation using strong ionization discharge[C].Mechanic Automation and Control Engineering (MACE)PP. 2094-2097. (2010).

7. Jiang L. Zhu R. Mao Y. Chen J. Zhang L. Conversion characteristics and production evaluation of styrene/o-xylene mixtures removed by DBD pretreatment. Int J Environ Res Public Health. 12(2): 1334-1350. (2015).

8. Guo Y. Liao X. Fu M. Huang H. Ye D. Toluene decomposition performance and NOx by-product formation during a DBD-catalyst process. J Environ Sci 28: 187-194. (2015). 